\title{
Nitroarene Carcinogen
}

National Cancer Institute

\section{Source}

National Cancer Institute. Nitroarene Carcinogen. NCI Thesaurus. Code C45182.

Potentially cancer-causing compound containing carbon and hydrogen in the form of an unsaturated, usually hexagonal, ring structure with one or more nitro-group(s), -NO2, attached to one or more aromatic ring(s). The compounds can be single ring, or double, triple, or multiple fused rings. These compounds are normally found in particulate emissions from many combustion sources, like diesel exhaust. (NCI05) 